Documentation et bibliothèques

DOCUMENTATION BIBLIOTHEQUES

\title{
Les services de recherche parlementaires
}

Parliamentary Research Services

\section{Los servicios de investigación parlamentaria}

\section{Hugh Finsten}

Volume 47, numéro 4, octobre-décembre 2001

Les bibliothèques parlementaires

URI : https://id.erudit.org/iderudit/1030376ar

DOI : https://doi.org/10.7202/1030376ar

Aller au sommaire du numéro

Éditeur(s)

Association pour l'avancement des sciences et des techniques de la documentation (ASTED)

ISSN

0315-2340 (imprimé)

2291-8949 (numérique)

Découvrir la revue

Citer cet article

Finsten, H. (2001). Les services de recherche parlementaires. Documentation et bibliothèques, 47(4), 153-157. https://doi.org/10.7202/1030376ar
Résumé de l'article

Les bibliothèques parlementaires, surtout celles calquées sur le modèle anglo-saxon, britannique ou américain, administrent des services d'études ou de recherche mis à la disposition des parlementaires et des organes intérieurs des Assemblées. L'auteur en présente ici une photo d'ensemble en insistant cependant sur la réalité vécue au Parlement fédéral canadien.
Tous droits réservés ( Association pour l'avancement des sciences et des techniques de la documentation (ASTED), 2001
Ce document est protégé par la loi sur le droit d'auteur. L’utilisation des services d'Érudit (y compris la reproduction) est assujettie à sa politique d'utilisation que vous pouvez consulter en ligne.

https://apropos.erudit.org/fr/usagers/politique-dutilisation/ 


\title{
Les services de recherche parlementaires
}

\author{
Hugh Finsten* \\ Bibliothèque du Parlement, Ottawa, Canada
}

Les bibliothèques parlementaires, surtout celles calquées sur le modèle anglo-saxon, britannique ou américain, administrent des services d'études ou de recherche mis à la disposition des parlementaires et des organes intérieurs des Assemblées. L'auteur en présente ici une photo d'ensemble en insistant cependant sur la réalité vécue au Parlement fédéral canadien.

\section{Parliamentary Research Services}

Parliamentary libraries, especially those based on the AngloSaxon, British or American models, manage research services for their members of parliament and other internal services. The author describes these services placing particular emphasis on the research service of the Canadian federal parliament.

\begin{abstract}
Los servicios de investigación parlamentaria
Las bibliotecas parlamentarias, particularmente aquellas calcadas del modelo anglosajón, británico o estadounidense, administran servicios de estudio o de investigación que se encuentran a disposición de los miembros del parlamento y las dependencias de dicha institución. El autor nos presenta aquí una fotografía grupal, tomando como caso de análisis la realidad observada en el parlamento federal canadiense.
\end{abstract}

Les organes d'information et de recherche des Assemblées législatives sont devenus indispensables aux législateurs du monde entier. Plusieurs Assemblées en sont dotées depuis bien des années et les pays moins développés sont toujours plus nombreux à en créer, souvent dans le cadre de programmes de collaboration internationale. Ces services s'inscrivent dans le processus de consolidation de la démocratie par le biais d'un soutien au pouvoir législatif.

II est facile de comprendre pourquoi ces services sont devenus aussi essentiels. Après tout, l'organe législatif d'un gouvernement doit disposer des ressources nécessaires pour assumer ses fonctions, par exemple adopter des lois, débattre des politiques du gouvernement, approuver les budgets et les dépenses de l'État, analyser les demandes de fonds que présentent les divers ministères chaque année et revoir les activités de ces derniers. De plus, les députés et les comités parlementaires proposent des projets indépendamment de ceux du gouvernement, amendent les projets de loi du gouvernement, rédigent des ébauches de politiques et formulent des jugements qui leur sont propres sur des questions dont est saisi le Parlement. En fait, les services d'information et de recherche des Assemblées législatives sont le pendant des ressources considérables dont dispose l'exécutif, mais n'en représenteront toujours qu'une fraction.

La politique et la législation, comme bien d'autres aspects du monde moderne, sont d'une complexité croissante. Le législateur est censé comprendre et interpréter tout ce qui se présente à lui, que ce soit dans le domaine fiscal, environnemental, monétaire ou constitutionnel. Pour être efficaces dans leurs fonctions législatives, les parlementaires doivent disposer de renseignements à jour, fiables, impartiaux et présentés dans un contexte pertinent.

Les députés reçoivent chaque jour un déluge de courrier de leurs électeurs, de groupes d'intérêt, de caucus de partis, de ministères, de sociétés privées et de groupes de pression qui cherchent tous à les influencer et à les presser d'appuyer ou de rejeter un projet de loi, une politique ou un point de vue particulier. Ils doivent donc disposer de leurs propres sources d'information et obtenir des renseignements objectifs, de manière à pouvoir se prononcer en toute indépendance sur les dossiers dont ils sont saisis.

Avec l'accessibilité universelle d'Internet, les sources d'information, voire l'information elle-même, ont connu une croissance exponentielle. Une grande part de l'information est d'excellente qualité même si certaines sources sont peu fiables. Les députés doivent donc être dotés de moyens pour se retrouver dans ce dédale d'information et distinguer ce qui est pertinent, exact et à jour de ce qui ne l'est pas.

Les services d'information et de recherche des Assemblées doivent être non partisans. Leur personnel est en fait au service de l'ensemble des députés qui peuvent ainsi, rapidement et sur un pied d'égalité, consulter avocats, économistes, spécialistes des sciences politiques, sociologues, scientifiques et autres experts. Ces services donnent suite aux demandes que leur présentent les députés après avoir consulté l'attaché de recherche et travaillent à un ensemble de publications générales sur les questions politiques intéressant les députés. Les parlementaires peuvent compter sur ces experts pour le type de recherche dont ils ont besoin sans craindre d'obtenir un point de vue personnel ou tendancieux.

II peut sembler, au premier coup d'œil, que ce sont les députés de l'opposition qui tirent le plus grand profit des servi-

\footnotetext{
* Pour rédiger le présent document, je me suis appuyé sur l'expérience de la Direction de la recherche parlementaire canadienne et sur des exemples de services qu'offrent d'autres entités de recherche parlementaires. Je suis reconnaissant à mes collègues de la Section des services de recherche et des bibliothèques des Assemblées législatives de 1'IFLA qui m'ont fourni des renseignements et des exemples. J'aimerais remercier, pour ses conseils extrêmement précieux, William Robinson du Congressional Research Service, un des chefs de file pour ce qui est de l'élaboration, de l'amélioration et du soutien des services de recherche dans le monde entier.
} 
ces de recherche des Assemblées, car les députés du parti au pouvoir peuvent utiliser plus librement les ressources du gouvernement en place; or, au Canada, ces derniers utilisent autant les services de recherche parlementaires. Ils y recourent pour mieux comprendre certaines questions complexes, pour élaborer des mesures législatives et des politiques indépendantes, pour contrer des projets et les arguments de l'opposition, etc. À Ottawa, les statistiques démontrent que les agents travaillent toujours en parts égales pour les deux catégories de députés.

\section{Établissement des services de recherche dans le monde}

Dans un certain nombre de pays du Commonwealth et aux États-Unis, les services de recherche ont émané de la bibliothèque parlementaire, prolongement naturel des livres, articles, documents et services de référence fournis par les bibliothèques. Aux États-Unis, la Bibliothèque du Congrès a créé le Legislative Reference Service en 1913; la recherche a pris forme en 1946, avec l'adoption d'une loi obligeant la Bibliothèque à employer des spécialistes chevronnés. En 1970, le service a pris le nom de Congressional Research Service (CRS) et est devenu le principal service de ce type du monde moderne. En Grande-Bretagne, c'est en 1946 que la Bibliothèque de la Chambre des communes a instauré un service de recherche. Les Parlements canadiens et australiens lui emboîtèrent le pas en 1965 et 1966 respectivement. Dans bien des pays européens comme la France, l'Allemagne, l'Italie et l'Espagne, les services parlementaires sont dotés depuis longtemps d'entités de recherche, mais, contrairement à leurs homologues britannique et américain, ces entités ne font pas partie de la bibliothèque des Assemblées législatives. Un certain nombre de nouvelles démocraties d'Europe de l'Est et d'Europe centrale, la Pologne et la Roumanie par exemple, ont établi en priorité des services de recherche parlementaires ${ }^{1}$. En Palestine, le Conseil législatif dispose d'un petit centre de recherche au service de ses membres. Ces services de recherche plus récents ont été mis sur pied indépendamment des bibliothèques des Assemblées législatives.
Pour ce qui est de la structure hiérarchique de ces services de recherche, il y a diverses variantes. Ce peut être un organisme distinct au sein du Parlement avec un chef qui relève du secrétaire général. Le service peut parfois être intégré à un organisme parlementaire existant, comme une bibliothèque ou un centre de documentation et d'information. Lorsqu'elles font partie des bibliothèques parlementaires, ces entités sont soit distinctes, soit combinées à un service d'information. Une autre option, qui est plutôt rare, consiste à établir ce service de recherche au sein d'un organisme ne faisant pas partie du Parlement (C'est ce que le Népal et l'Éthiopie envisagent de faire).

À une conférence tenue à Ottawa en 1998, l'évolution et le fonctionnement de plusieurs services de recherche d'Assemblées bien établies ont été comparés ${ }^{2}$. II est intéressant de noter que même si ces services se sont développés indépendamment les uns des autres et n'ont eu que de rares contacts au fil des années, ils offrent des services et des produits presque identiques et leur organisation ainsi que les qualifications professionnelles de leur personnel se comparent. II semble donc que les exigences et les impératifs des Parlements du monde entier ainsi que la nature de l'information dont ils ont besoin pour assumer leurs fonctions, et ce, malgré les différences culturelles et historiques, se ressemblent beaucoup.

\section{Principes de base}

Les services sont «apartisans »: le personnel sert les parlementaires quels que soient leur parti ou leur idéologie, de la même façon et par ordre d'arrivée des demandes. Les résultats des recherches des services sont objectifs: le personnel n'encourage aucun point de vue particulier, que ce soit d'ordre personnel ou politique. Les services sont confidentiels: le personnel ne peut divulguer le nom de la personne pour laquelle il travaille ou la teneur des recherches menées pour un député. Dans certains services, les documents ne sont diffusés qu'avec le consentement du député pour lequel ils ont été préparés. Dans d'autres, il faut attendre un certain temps avant qu'un document produit pour un député en particulier puisse être publié.

\section{Les services de recherche dans les Parlements}

\section{Services types}

Un service de recherche centralisé est constitué d'une équipe de consultants qui sont à la disposition des parlementaires. Dans les Parlements dotés de fonds limités, un service centralisé est un moyen économique de fournir à l'ensemble des députés la possibilité de consulter des spécialistes. En revanche, dans bon nombre de Parlements établis de longue date, les députés ont accès à un service de recherche et disposent également de budgets leur permettant d'engager leur propre personnel de recherche. Des experts en procédure, en droit ou en rédaction de projets de loi ainsi que les attachés de recherche du parti peuvent également apporter leur concours.

La plupart des services de recherche proposent le même genre de produits et de services, quelle que soit leur importance.

Aux députés, ils fournissent une aide personnalisée conformément aux besoins exprimés. Un tel service constitue souvent la raison première de la création d'un service de recherche, c'est-à-dire aider les députés et donc améliorer la qualité des débats et le travail du Parlement. Tous les députés peuvent consulter un spécialiste en tout temps. Les services fournis vont de la préparation d'études approfondies répondant aux besoins de l'intéressé pour des séances d'information, aux renseignements donnés par téléphone jusqu'à la production de notes d'information succinctes, d'études de fond sur des questions d'actualité et de publications expliquant la politique ou les mesures législatives du gouvernement.

De nombreux services de recherche notent que les parlementaires demandent des réponses précises et concises dont ils peuvent prendre connaissance rapidement plutôt que des recherches approfon-

1. Robinson, William H and Raymond Gastelum. 1998. Parliamentary libraries and research services in Central and Eastern Europe. Munich : K.G.Saur. (IFLA Publication 87)

2. Comparative legislative research services seminar. 1998. Ottawa : Bibliothèque du Parlement. 
dies, détaillées et plus formelles. Dans de nombreux pays, les parlementaires réclament de plus en plus des réponses orales. Ils attendent des réponses plus approfondies lorsqu'ils veulent comprendre dans le détail un dossier particulier. Les publications à diffusion générale peuvent également répondre à des demandes de renseignements détaillés sur une question particulière.

\section{Recherches, publications, analyses}

Le type de recherches des députés varie selon les questions et les centres d'intérêt de ces derniers. Elles peuvent porter sur la législation en matière de stupéfiants dans divers pays, sur des articles de lois nationales à modifier pour que prenne effet une politique ou une modification particulière, sur des changements aux impôts payés par certaines catégories de contribuables par suite d'une variation des taux d'imposition, sur les effets environnementaux d'un incendie dans un entrepôt de pneus, sur une question complexe émanant d'un électeur ou sur des notes en vue d'une allocution en Chambre, lors d'une conférence ou d'une réunion avec des électeurs ou des groupes de pression. II peut falloir quelques heures, quelques jours, parfois plusieurs semaines ou mois pour fournir une réponse selon la complexité de la question, la disponibilité du personnel et les exigences du député.

Les publications à diffusion générale sont des documents rédigés par le personnel et distribués, sur demande, à l'ensemble des députés. Ils portent sur des dossiers dont la Direction sera saisie et sur de nouvelles questions qui pourront se poser. Ces documents permettent de gérer la charge de travail, car ils peuvent répondre à un grand nombre de demandes présentées séparément et, par conséquent, permettre d'économiser temps et ressources. Ainsi, le personnel peut se concentrer sur d'autres dossiers ou des questions particulières relevant d'un sujet plus général et préparer des notes personnalisées à l'intention d'un député. II ne leur est donc pas nécessaire de rédiger un nouveau document pour chaque demande reçue.

Les publications prennent diverses formes. Les études générales sont des études approfondies sur une question particulière destinées aux députés désirant connaître à fond un sujet. Les bulletins d'actualité sont de brefs commentaires descriptifs sur des questions mis à jour régulièrement pour que les députés disposent de données récentes sous une forme concise ; ils peuvent comprendre un exposé des mesures prises par le Parlement ainsi qu'une chronologie des faits marquants et une bibliographie sélective à l'intention de ceux qui désirent approfondir le sujet. Les résumés (ou condensés) législatifs donnent des renseignements sur les projets de loi, leur historique et leur contexte et peuvent comprendre une analyse ainsi que des commentaires donnés article par article. Enfin, les documents de recherche de quelques pages fournissent un aperçu d'une question à l'intention des députés qui n'ont pas besoin d'une étude détaillée et approfondie d'un sujet.

Les services en ligne se développant rapidement, ces publications d'ordre général sont souvent disponibles en format électronique pour que le député puisse les consulter sur-le-champ et les imprimer directement sans avoir à communiquer avec le service de recherche. D'autres publications ont été créées uniquement en format électronique et contiennent des liens menant à des sites Web complémentaires.

Un service spécial est offert aux députés qui désirent rédiger des projets de loi d'initiative parlementaire. Bien que la rédaction véritable du projet de loi soit généralement le fait des conseillers législatifs, le service de recherche peut s'occuper du travail de préparation nécessaire. II s'agit notamment d'obtenir des renseignements généraux concernant le sujet et le contexte dont il faut tenir compte (considérations d'ordre constitutionnel, législation existante pouvant être touchée et devant être modifiée et projets de loi de même nature déjà présentés, etc.). Le député dispose ainsi des données dont il a besoin pour répondre aux questions techniques concernant le projet de loi, données dont il pourra se servir pour ses discours en Chambre, devant les comités et ailleurs. Ce travail aide également les rédacteurs à comprendre l'intention du député et la législation à modifier. Les avocats, les attachés de recherche et les rédacteurs doivent souvent collaborer étroitement.

Lorsque la demande émane des comités, les services de recherche y répondent comme ils le feraient pour un député. II arrive que certains comités demandent des analyses plus complètes et plus approfondies que ne l'exigent en général les dépu- tés, ce qui prend forcément plus de temps. À Ottawa, Toronto et dans le Rajya Sabha, le Conseil des États ou Chambre haute de l'Inde, les services de recherche mettent leurs spécialistes à la disposition des comités.

Pour les délégations parlementaires, les services de recherche préparent des documents et fournissent d'autres informations concernant les points à l'ordre du jour des réunions régionales et internationales des associations parlementaires ou des rencontres bilatérales ou multilatérales de parlementaires. Bien souvent, ils fournissent des renseignements généraux sur le pays où se tient la réunion, notamment sur le gouvernement, la situation politique et économique, ainsi que des statistiques sur le commerce et d'autres questions bilatérales. Un membre du service peut également accompagner, en qualité de spécialiste, la délégation à ces réunions et conférences.

\section{Mémoire de l'institution}

La mémoire de l'institution est souvent un aspect oublié bien qu'elle ait un rôle important à jouer dans le cadre législatif. Dans de nombreux pays, le roulement des députés, d'une élection à l'autre, est élevé. Il est donc important pour que le Parlement fonctionne efficacement qu'il y ait une mémoire institutionnelle. C'est là qu'intervient le personnel du Parlement: pour les membres du service de recherche, il s'agit de connaître les questions de fond et les projets de loi déjà discutés. Cela est particulièrement vrai pour les comités où la permutation des membres est encore plus fréquente d'une présidence à l'autre et d'un comité à l'autre. Ceux qui ont été affectés à un comité en particulier sont donc au fait de ses activités: les études et les discussions qui y ont été tenues occupent donc une place prépondérante dans le travail effectué pour ce dernier. Les écrits ne sont qu'une facette de cette mémoire. Parce que le personnel qui a participé à une étude a pris des notes et se souvient des travaux effectués, il est souvent possible d'actualiser le travail déjà fait sans être obligé de recommencer à zéro.

\section{Services supplémentaires}

Comme nous l'avons déjà mentionné, les Assemblées législatives à Ottawa et à Toronto et le Rajya Sabha, en Inde, mettent 
leur personnel - attachés de recherche ou équipe multidisciplinaire - à la disposition des comités parlementaires. À Ottawa, un criminologue et deux avocats travaillent pour le Comité de la justice de la Chambre des communes; un économiste, un avocat et un scientifique sont affectés au Comité de l'industrie et deux économistes et un avocat travaillent pour le Comité sénatorial des banques.

Une autre possibilité est le détachement de personnel auprès des comités soit pour les aider à étudier des questions particulières, soit pour des périodes déterminées (Australie). L'attaché de recherche travaille exclusivement pour le comité durant cette période. Le CRS fournit aux comités un appui et une aide soutenus en fournissant des spécialistes au gré des besoins.

Le type de travail entrepris à la demande des comités varie selon les besoins et les exigences de chacun. II peut s'agir:

d'aide pour rédiger un plan d'action et un programme pour les audiences du comité;

de la préparation d'une liste de témoins, incluant des renseignements généraux sur chaque groupe ou particulier;

de la rédaction de notes d'information sur des sujets particuliers;

- de l'élaboration de notes d'information en vue d'audiences de comités comprenant: biographies des témoins, résumés des mémoires et questions à poser éventuellement;

- de la présentation orale de notes d'information aux comités, de consultations des députés, conseils et recommandations;

de la préparation d'études approfondies sur des questions particulières;

de l'élaboration de documents sur des dossiers et la présentation des options qui en découlent;

de la collecte et de l'analyse de renseignements;

de la constitution de dossiers d'information comprenant souvent des graphiques permettant d'établir des comparaisons, des commentaires sur des questions ou des témoignages, des documents d'origine et des articles de périodiques;

E de la préparation d'ébauches de rapports;

de la rédaction de communiqués de presse;

- de la tenue de séances d'information avant publication.
Par ailleurs, le personnel affecté aux comités continue de répondre aux demandes des députés et d'autres comités et prépare également des publications. II doit souvent mener de front plusieurs études pour respecter les délais.

\section{Séances et ateliers d'information}

II s'agit de sensibiliser les députés et leur personnel au rôle des services de recherche et d'information, d'informer les députés des nombreuses ressources dont ils peuvent se prévaloir directement et d'accroître l'efficacité des services de recherche et d'information. Le personnel de recherche participe à ces séances et ateliers afin que les parlementaires se familiarisent avec eux ainsi qu'avec les ressources mises à leur disposition. Ainsi, le personnel parlementaire peut obtenir des conseils sur la façon de trouver des renseignements lui permettant par la suite de faire le travail lui-même. II peut s'agir, notamment de la manière de s'y retrouver dans les lois et les règlements, de répondre aux questions des électeurs sur des sujets d'ordre fiscal, sur les avantages sociaux, sur l'immigration et sur les réfugiés, ou encore d'utiliser des documents de référence clé. Les ateliers sur les politiques publiques dans des domaines particuliers qui sont tenus par des spécialistes ou des experts externes fournissent des renseignements généraux en vue de discussions et de débats. Ces ateliers permettent au service de recherche d'assumer son rôle naturel, à savoir faire la promotion du service et informer les députés et leur personnel à la manière d'une « université du Parlement ».

Après des élections, de nombreux services d'études offrent des programmes destinés aux nouveaux députés. Le Congressional Research Service fournit des sessions périodiques d'information sur les politiques publiques à l'intention des nouveaux membres du Congrès et des comités qui le désirent. Ces sessions sont financées par des fondations privées et sont normalement tenues à l'extérieur du Parlement. Des experts d'un peu partout au pays sont invités à faire le point à l'intention des membres du Congrès sur les questions de politiques importantes et à susciter des débats.

\section{Experts externes}

L'Australie et les États-Unis recourent très souvent à des spécialistes pour de courtes périodes. Il est aussi possible d'engager des fonctionnaires retraités, des avocats, des universitaires, des employés de ministères et d'autres experts sur une base contractuelle. Le service peut ainsi libérer son personnel pour qu'il puisse se perfectionner. Ottawa a déjà fait appel à des spécialistes externes pour la préparation de documents dans le cadre de son programme de publications.

Le Parlement canadien recourt à des spécialistes de l'extérieur pour les travaux de ses comités. Ces derniers s'ajoutent au personnel de la Direction et peuvent faire profiter les comités de leur expérience et de leurs connaissances pour des études importantes. La Direction s'est également entendue avec certains ministères pour obtenir des détachements de personnel. La meilleure solution consiste à intégrer les consultants à l'équipe de recherche afin de faire profiter cette dernière de leurs connaissances et de leur expérience d'un sujet particulier; l'équipe étant davantage axée sur le contexte parlementaire. L'Institut parlementaire tchèque et le Sénat polonais font souvent appel à des contractuels.

\section{Publications électroniques}

Lorsque les députés et le personnel ont facilement accès à des outils électroniques, notamment Internet, les services de recherche et d'information conçoivent leurs produits en conséquence. Les Electronic Briefing Books du CRS, les $E$ Briefs australiens, les Capsules d'information canadiennes et les Issue Gateways torontois en sont des exemples. II peut s'agir de brèves analyses établissant un contexte, de liens menant à divers interlocuteurs, d'articles de presse choisis, de documents venant de groupes de réflexion et d'autres rapports ou documents de fond. Ces produits électroniques sont conçus pour servir de point de contact avec les préoccupations de l'heure.

\section{Condensés de projets de loi}

II s'agit d'analyses de projets de loi présentés par le gouvernement. Au Canada, la rédaction des résumés législatifs a vu le jour au milieu des années 1990 à la suggestion d'un parlementaire (qui a dit 
que les employés n'écoutaient pas les parlementaires!). Ils fournissent des renseignements généraux sur le projet de loi, un résumé et une analyse des plus importants articles ainsi qu'un commentaire de spécialistes externes. Ces documents sont très appréciés. À Ottawa, chaque fois qu'un comité étudie un projet de loi, un résumé législatif est distribué à ses membres. II est vrai que le gouvernement transmet régulièrement des documents expliquant les mesures législatives qu'il se propose d'adopter, mais les résumés législatifs, comme tous les documents de recherche, ne proviennent pas d'une source partisane et les députés peuvent se fier à leur objectivité.

\section{Analyse budgétaire}

Dans la Chambre basse polonaise, une entité distincte du bureau de recherche traite de l'analyse budgétaire et fournit une aide directe aux comités chargés des questions de politique économique, du budget et des finances. Au Parlement slovaque, le service de recherche fournit, depuis sa création au début des années 1990, des services limités d'analyse budgétaire. En Suède, le service a établi un bureau du budget en 1997 en raison de la mise en place de nouvelles procédures budgétaires par le gouvernement et de la multiplication des demandes de calcul découlant du budget émanant des députés et des secrétariats des partis. En Norvège, le bureau du budget parlementaire, créé en 1998, est constitué de deux économistes. Au moment de la mise sur pied du service de recherche, en 1999, il a été décidé que ce dernier pourrait utiliser ces économistes si ceux-ci étaient disponibles. L'inverse est également vrai.

\section{Recherche sur le terrain}

$\mathrm{Au}$ Japon, le bureau de recherche et de référence des Assemblées législatives offre un service unique: une dizaine d'analystes se déplacent dans le pays chaque année pour mener des recherches sur le terrain. Ces recherches peuvent porter, par exemple, sur l'impact d'une base militaire sur l'environnement ou de l'effet de la récession sur l'industrie locale. Les analystes interrogent les personnes intéressées, notamment des représentants du gouvernement local et des chefs de groupes de citoyens et ils réunissent des documents auxquels les attachés de recherche n'auraient pas autrement accès. Les résultats de ces enquêtes sont diffusés auprès des députés par l'entremise des publications du bureau.

\section{Une ressource fondamentale}

Nous avons passé brièvement en revue la raison d'être et l'évolution des services de recherche parlementaires ainsi que la nature des produits et services qu'ils offrent couramment. Les parlementaires ont besoin d'information pour assumer leurs fonctions en toute connaissance de cause. Les services de recherche sont donc une ressource fondamentale pour les aider à comprendre les dossiers dont ils sont saisis et à avoir une vue globale des situations. Les services de recherche et d'information des Assemblées législatives représentent une source d'information et d'analyse à la fois économique, impartiale et fiable grâce à l'équipe de professionnels dont ils sont constitués. L'expansion de ces services dans les Parlements des pays développés et en développement témoigne de leur importance dans les sociétés démocratiques. 